\title{
Physical activity in daily life is associated with lower adiposity values than doing weekly sports in Lc65+ cohort at baseline
}

Nadia Danon-Hersch ${ }^{*}$ and Brigitte Santos-Eggimann ${ }^{*}$

\begin{abstract}
Background: Overweight and obesity prevalence is the highest at age 65-75 years in Lausanne (compared with younger classes). We aimed to describe 1) eating habits, daily physical activity (PA), and sports frequency in community-dwelling adults aged 65-70, 2) the links of these behaviors with socio-economic factors, and 3) with adiposity.
\end{abstract}

Methods: Cross-sectional analysis of Lc65+ cohort at baseline, including 1260 adults from the general population of Lausanne aged 65-70 years. Eating habits (8 items from MNA) and PA (sports frequency and daily PA: walking and using stairs) were assessed by questionnaires. Body mass index (BMI), supra-iliac (SISF), triceps skin-folds (TSF), waist circumference (WC), and WHR were measured.

Results: Prevalence of overweight (BMl $\left.25.0-29.9 \mathrm{~kg} / \mathrm{m}^{2}\right)$, obesity $\left(\mathrm{BMI} \geq 30.0 \mathrm{~kg} / \mathrm{m}^{2}\right)$, and abdominal obesity was $53 \%, 24 \%$, and $45 \%$ in men; $35 \%, 23 \%$, and $45 \%$ in women.

Intake of fruits or vegetables (FV) $\geq$ twice/day was negatively associated with male sex (prevalence $81 \%$ versus $90 \%$, chi-square $P<0.001$ ). The proportion avoiding stairs in daily life was higher among women (25\%) than among men (20\%, chi-square $P=0.003)$.

In multivariate analyses among both sexes, eating FV, using stairs in daily life ("stairs"), and doing sports $\geq$ once/week were significantly negatively associated with financial difficulties (stairs: $\mathrm{OR}=0.54,95 \% \mathrm{Cl}=0.40-0.72$ ) and positively with educational level (stairs: $\mathrm{OR}=1.68,95 \% \mathrm{Cl}=1.17-2.43$ for high school).

For all five log-transformed adiposity indicators in women, and for all indicators except SISF and TSF in men, a gradual decrease in adiposity was observed from category "no stairs, sports < once/week" (reference), to "no stairs, sports $\geq$ once/week", to "stairs, sports < once/week", and "stairs, sports $\geq$ once/week" (for example: WC in men, respectively: $\beta=-0.03,95 \% \mathrm{Cl}=-0.07-0.02 ; \beta=-0.06,95 \% \mathrm{Cl}=-0.09--0.03 ; \beta=-0.10,95 \% \mathrm{Cl}=-0.12--0.07)$.

Conclusions: In this population with high overweight and obesity prevalence, eating FV and PA were strongly negatively associated with financial difficulties and positively with education. Using stairs in daily life was more strongly negatively associated with adiposity than doing sports $\geq$ once/week.

Keywords: Obesity, Adiposity, Eating habits, Daily physical activity, Stairs, Sports

\footnotetext{
* Correspondence: nadiadanon@yahoo.fr; Brigitte.Santos-Eggimann@chuv.ch

Institute of Social and Preventive Medicine (IUMSP), University of Lausanne

Hospital Center, Route de la Corniche 10, Lausanne 1010, Switzerland
}

\section{Biomed Central}

(c) 2013 Danon-Hersch and Santos-Eggimann; licensee BioMed Central Ltd. This is an open access article distributed under the terms of the Creative Commons Attribution License (http://creativecommons.org/licenses/by/2.0), which permits unrestricted use, distribution, and reproduction in any medium, provided the original work is properly cited. 


\section{Background}

In Switzerland, the prevalence of overweight and obesity has increased in all age groups between 1992 and 2007 [1]; in the city of Lausanne, it was the highest in age group 65-75 years in 2005, compared to younger groups, reaching a total prevalence of $73 \%$ among men, 53\% among women [2]. This difference by age was also observed in the Swiss Health Survey 2012, a nationwide study using self-reported height and weight: the prevalence with body mass index $(\mathrm{BMI}) \geq 25 \mathrm{~kg} / \mathrm{m}^{2}$ reached $56 \%$ at age 65-74 years [3]. Overweight and obesity are important risk factors for chronic diseases and disability [4]. The youngest old deserve special attention because while the risks of obesity, chronic diseases, and disability are still present at this age, this population bears the additional risk of malnutrition, frailty [5], and sarcopenia [6,7]. In obese older adults, improving dietary habits and increasing physical activity (PA) appear to be the most effective strategies in helping to decrease body weight and improve function and survival $[4,8,9]$. PA has the potential to reduce the risks of both obesity [10] and sarcopenia [11]. To our knowledge, most randomized controlled trials focus on increasing the frequency of sports sessions, while increasing PA in everyday life in the long term is less often the target. In addition, participants are rarely asked in observational studies on PA if they usually climb stairs in their daily life.

In this context, it is important to have a more precise picture of how persons of the general population aged 65 to 70 years eat and expend energy, and how these behaviors are affected by socioeconomic factors. According to our literature review, the associations of eating habits and PA with socioeconomic position and adiposity have not been explored in detail in this age range. A positive link between socioeconomic level and PA has been described [12,13] in older adults. Concerning dietary habits, a Swiss study (CoLaus) in the same city has recently observed that the nutritional recommendations were only slightly followed in the general population (age range 40-82 years at follow-up) [14]: only 39\% and $7 \%$ complied with the Swiss recommendations for fruit ( $\geq 2$ /day) and vegetables ( $\geq 3$ /day). Many studies in young adults have observed that nutrition knowledge and compliance with dietary guidelines have positive relationships with female sex, marital situation [15], high socioeconomic position [12], and higher fast food prices [16]. In Switzerland, a link between overweight and obesity and low education status has been observed in four cross-sectional National health surveys using representative samples of the Swiss population aged 18-102 years [1]; however differences in financial resources were not taken into account in this analysis. Changes in body composition are observed with advancing age $[8,17]$, highlighting the need to monitor adiposity trends with several indicators, including BMI and waist circumference (WC) [18]. Although sophisticated methods exist for exploring adiposity, simple measures such as anthropometric indicators are useful for routine clinical practice and public health surveillance.

The present article aims to identify a hierarchy in prevention efforts: diet, versus PA; and daily PA versus sports frequency. We hypothesized that the youngest old with more or less healthy eating habits, daily PA, and sports frequency would have different anthropometric adiposity values. Our aim was to describe 1) eating habits, daily physical activity (PA), and sports frequency in community-dwelling adults aged 65 to 70,2$)$ the links of each of these behaviors with socioeconomic factors, and 3) with adiposity.

\section{Methods}

\section{Study design and participants}

The Lausanne cohort Lc65+, a study of the manifestations, determinants and outcomes of frailty [19], recruited individuals aged $65-70$ in 2004, stemming from a representative sample of the general non-institutionalized population living in Lausanne (random sample from the list of all inhabitants given by the Population Office). The design of Lc65+ has already been described [19]. The ethics committee of the Faculty of Biology and Medicine of the University of Lausanne has approved the protocol. All participants provided written informed consent. Persons living in institutions or unable to respond because of advanced dementia were excluded. Of the 3,056 people who were initially mailed questionnaires, 2,096 (69\%) replied, of whom 1,564 (75\%) agreed to participate [19]. Overall, nonparticipants had demographic characteristics similar to those of participants [19]; only $8 \%$ of those refusing to participate attributed their refusal to poor health, and 58\% had "a general reluctance to participate in any survey". Of the 1,564 respondents to the initial questionnaire, 1,524 (97.4\%) were still eligible, and 1,422 (93.3\%) participated in the baseline assessment in 2005 [5,19]. This report is based on a cross-sectional analysis of the cohort at baseline (2004-5). Eating habits [20] and PA could be influenced by cognitive impairment. Therefore, 162 persons with Mini-mental State Score $<24$ [21] $(\mathrm{n}=49)$ or missing $(\mathrm{n}=113)$ were excluded and 1,260 participants remained in this analysis.

In 2004, all participants completed a questionnaire sent at home; in 2005, they underwent the assessment at the study center with an interview, measurements, and performance tests conducted by trained medical assistants.

\section{Eating habits and physical activity}

A selection of 8 items stemming from the Mini Nutritional Assessment (MNA) [22], and describing eating habits was asked to the study population. The MNA is a 
widely used tool for assessing the risk of malnutrition in older adults [22].

The following MNA questions were selected (Tables 1 and 2): A) How many full meals do you eat daily? 1) 1 meal; 2) 2 meals; and 3) 3 meals. B) Do you consume at least one serving of dairy products per day? 1) yes; 2) no. C) Do you consume at least two servings of legumes or eggs per week? 1) yes; 2) no. D) Do you consume meat, fish or poultry every day? 1) yes; 2) no. E) Do you consume two or more servings of fruits or vegetables per day? 1) yes; 2) no. F) Do you have a loss of appetite? 1) yes, severe; 2) yes, moderate; 3) not at all. G) How many drinks do you consume per day (water, juice, coffee, tea, milk, wine, beer, soup...)? 1) less than 3 glasses (1 glass $=2 \mathrm{dl}$.); 2) 3 to 5 glasses; 3) more than 5 glasses. H) Do you view yourself as being well fed, as having no nutritional problem? 1) severe malnutrition; 2) does not know or moderate malnutrition; 3) no nutritional problem. The 8 items' distributions are shown in Table 1.

Since the MNA does not include any quantitative assessment of total energy intake, only three questions entailing indirect (however incomplete) information about energy intake were kept for multivariate analyses of the associations between diet and PA and adiposity indicators. In order to have a simple binary variable summarizing protein intake in these analyses, "sufficient protein intake" was defined if the participant reported eating meat, fish or poultry every day, or as an alternative, if he consumed dairy products $\geq$ once a day and eggs or leguminous plants $\geq$ twice/week.

PA was assessed with 2 main questions, described hereafter: daily PA and sports frequency (Table 3 ). This measurement of PA based on two questions has been adapted from the Monitoring of Trends and Determinants in Cardiovascular Disease Physical Activity Questionnaire $[23,24]$ to suit activity patterns of individuals aged $65-70$. In order to compare daily PA and sports frequency with respect to their association with adiposity, a four-category variable named "Daily PA and sports" combining both variables was created: "daily PA and sports" is a combination of a 4-category ordered variable (daily PA, further dichotomized around use, versus avoidance of stairs and loads) and a binary variable (sports frequency): A) Daily PA: "Which statement best describes your current daily physical activity? 1) I am sitting or lying most of the time and I am not moving much; 2) I often walk, but I avoid taking stairs and carrying loads; 3) I often walk and I take stairs, I carry light loads; and 4) I make an important physical effort, I often carry heavy loads." In the Figure 1 and in Table 4, categories 1) and 2) of daily PA are aggregated and labeled "No stairs"; categories 3) and 4) are aggregated and labeled "Stairs". B) Sports frequency: "How often do you play sports for at least 20 minutes (for example, gymnastics, tennis, running, football, biking...)? <1x/week; versus $\geq 1 /$ week." "No sport" is the label for sports frequency $<1 \times /$ week in the Figure 1 and in Table 4 . "Sports weekly" is the label for sports $\geq 1 \times /$ week. Therefore, the 4 categories of variable "Daily PA and sports" are: I) no stairs, no sport, II) no stairs, but sports weekly, III) stairs, but no sport, and IV) stairs and sports weekly.

\section{Adiposity indicators}

Height, weight, supra-iliac skin-fold (SISF), triceps skinfold (TSF), waist (WC), and hip circumferences were measured without shoes. Weight was assessed with a digital SECA scale. The standard procedure recommended in NHANES III [25] was followed. WC was measured at the level midway between the lowest rib and the highest point of the iliac crest. Hip circumference was recorded as the maximum circumference over the buttocks. Skin-folds were measured with a GPM ${ }^{\circ}$ caliper on the dominant side. Overweight and obesity were defined according to body mass index (BMI $=25.0$ $29.9 \mathrm{~kg} / \mathrm{m}^{2}$ and $\geq 30.0 \mathrm{~kg} / \mathrm{m}^{2}$ ). Abdominal obesity was considered if WC was $\geq 102 \mathrm{~cm}$ for men and $\geq 88 \mathrm{~cm}$ for women [26].

\section{Other Covariates}

Potential confounders of the diet-adiposity relationship or the PA-adiposity association are shown in Table 1 and adjusted for in multivariate models (Tables 3 and 4): age, living arrangement (living alone, 0/1), financial difficulties, symptoms of depression, education, and smoking status are known to be associated with eating habits $[15,27,28]$, PA (as independent variables) $[12,29]$, and adiposity (as the dependent variable) [8]. The educational level entitled "high school or more" includes high school, professional school, or university. Living alone was assessed by the question: "With how many persons are you currently living? 1) I am living alone; 0) I am living with ... persons". Financial difficulties was a variable of interest for objective 2 (the cross-sectional association between eating habits, PA, and socio-economic factors), and a potential confounder for objective 3. It is defined in the footnotes of Tables 1, 2, 3, and 4. Current symptoms of depression were considered for the participants who had answered "yes" to at least one of two screening questions [30]: "During the past month have you often been bothered by feeling down, depressed, or hopeless?" and "During the past month have you often been bothered by little interest or pleasure in doing things?" Since eating habits, PA, and adiposity indicators differ between men and women [31,32], all analyses are stratified by sex.

\section{Statistical analyses}

Results were expressed as absolute numbers and percentages. Bivariate comparisons were performed using the chisquare test or Fisher exact test for categorical variables. 


\begin{tabular}{|c|c|c|c|}
\hline & Men & Women & $x^{2}$ \\
\hline & $N=519$ & $N=741$ & $P$-value \\
\hline & N (\%) & N (\%) & \\
\hline \multicolumn{4}{|l|}{ OBESITY PREVALENCE } \\
\hline Body mass index category: & & & $<0.001$ \\
\hline Underweight $\left(\mathrm{BMl}<18.5 \mathrm{~kg} / \mathrm{m}^{2}\right)$ & $0(0.0)$ & $19(2.6)$ & \\
\hline Normal weight (BMl 18.5-24.9 kg/m²) & $120(23.3)$ & $290(39.2)$ & \\
\hline Overweight (BMI 25.0-29.9kg/m²) & $271(52.6)$ & $260(35.2)$ & \\
\hline Obesity $\left(\mathrm{BMI} \geq 30.0 \mathrm{~kg} / \mathrm{m}^{2}\right)$ & $124(24.1)$ & $170(23.0)$ & \\
\hline Abdominal obesity (waist circumference: WC $\geq 102 \mathrm{~cm}$ in men, $\geq 88 \mathrm{~cm}$ in women) & $228(44.6)$ & $334(45.3)$ & 0.823 \\
\hline WC-defined normal weight obesity (BMl $<25 \mathrm{~kg} / \mathrm{m}^{2}$ and $W C \geq 102 \mathrm{~cm}$ in men, $\geq 88 \mathrm{~cm}$ in women) & $0(0.0)$ & $16(2.2)$ & $<0.001^{* *}$ \\
\hline WHR-defined normal weight obesity (BMl $<25 \mathrm{~kg} / \mathrm{m}^{2}$ and WHR $\geq 66$ th gender-specific percentile) & $12(2.3)$ & $37(5.0)$ & $0.017 * *$ \\
\hline \multicolumn{4}{|l|}{ EATING HABITS } \\
\hline \multicolumn{4}{|l|}{ Questions from the MNA: } \\
\hline Number of meals per day* & & & 0.177 \\
\hline 1 meal/day & $33(6.4)$ & $60(8.1)$ & \\
\hline 2 meals/day & $204(39.4)$ & $257(34.8)$ & \\
\hline 3 meals/day & $281(54.3)$ & $422(57.1)$ & \\
\hline Meat, fish or poultry everyday & $265(51.3)$ & $343(46.4)$ & 0.087 \\
\hline Dairy products $\geq$ once a day & $425(82.1)$ & $643(86.8)$ & 0.021 \\
\hline Eggs or leguminous plants $\geq$ twice/week & $254(49.0)$ & $350(47.2)$ & 0.529 \\
\hline "Sufficient protein intake" & $375(72.5)$ & $514(69.5)$ & 0.239 \\
\hline Fruit or vegetables $\geq$ twice/day & $417(80.5)$ & $666(90.1)$ & $<0.001$ \\
\hline Drinks per day & & & 0.223 \\
\hline$<3$ glasses ( 1 glass $=2$ dl. $)$ & $40(7.7)$ & $58(7.8)$ & \\
\hline 3 to 5 glasses & $199(38.4)$ & $250(33.7)$ & \\
\hline$>5$ glasses & $279(53.9)$ & $433(58.4)$ & \\
\hline Loss of appetite & & & 0.627 \\
\hline Yes, severe & $1(0.2)$ & $4(0.5)$ & \\
\hline Yes, moderate & $25(4.8)$ & $35(4.7)$ & \\
\hline Not at all & $492(95.0)$ & $701(94.7)$ & \\
\hline Self-perception of nutrition & & & 0.708 \\
\hline Severe malnutrition & $0(0.0)$ & $0(0.0)$ & \\
\hline Doesn't know/moderate malnutrition & $21(4.1)$ & $27(3.6)$ & \\
\hline No nutritional problem & $497(96.0)$ & $714(96.4)$ & \\
\hline \multicolumn{4}{|l|}{ PHYSICAL ACTIVITY (PA): } \\
\hline Daily PA: & & & 0.003 \\
\hline Sitting or lying most of the time & $39(7.5)$ & $37(5.1)$ & \\
\hline Often walking, but avoids stairs and loads & $66(12.8)$ & $144(19.7)$ & \\
\hline Often walking and using stairs, carrying light loads & $373(72.2)$ & $512(69.9)$ & \\
\hline Important physical activity, carries heavy loads & $39(7.5)$ & $40(5.5)$ & \\
\hline Sports ( $\geq 20$ minutes) frequency: & & & 0.236 \\
\hline Less than once a week & $246(49.3)$ & $359(51.9)$ & \\
\hline Once or twice a week & $154(30.9)$ & $222(32.1)$ & \\
\hline Thrice a week or more & $99(19.8)$ & $111(16.0)$ & \\
\hline
\end{tabular}


Table 1 Obesity, eating habits and physical exercise in 2004-2005 (Continued)

\begin{tabular}{|c|c|c|c|}
\hline \multicolumn{4}{|l|}{ ADJUSTMENT VARIABLES: } \\
\hline Living alone (0/1) & $96(18.5)$ & $346(46.7)$ & $<0.001$ \\
\hline Financial difficulties $(0 / 1)^{\ddagger}$ & $123(23.7)$ & $207(27.9)$ & 0.092 \\
\hline Current symptoms of depression & $97(19.0)$ & $201(27.7)$ & $<0.001$ \\
\hline Education & & & $<0.001$ \\
\hline Basic compulsory & $82(15.9)$ & $218(29.5)$ & \\
\hline Apprenticeship & $225(43.5)$ & $274(37.1)$ & \\
\hline High school or more & $210(40.6)$ & $246(33.3)$ & \\
\hline Current smoking ${ }^{\S}$ & $119(23.2)$ & $143(19.5)$ & 0.112 \\
\hline
\end{tabular}

Categorical variables are given in numbers (\%); the chi-square $P$-value compares proportions across sexes.

**Fisher's exact test.

*Instructions given to interviewers: a breakfast includes a drink and one solid food item. A lunch or dinner meal needs to include a source of proteins, starchy food (feculent), and fruit or vegetables. It can be warm or cold. A sandwich is not considered as a meal.

'In order to have an approximate summary measure for protein intake, "Sufficient protein intake" was defined if the participant reported eating meat, fish or poultry every day, or as an alternative, if he consumed dairy products $\geq$ once a day and eggs or leguminous plants $\geq$ twice/week.

${ }^{\ddagger}$ Financial difficulties were considered if any of the following criteria was fulfilled: 1) current income clearly lower than others, 2) sometimes difficulty to make ends meet, 3) subsidy for health insurance, or 4) complementary subsidy (from old age insurance).

${ }^{\S}$ Participants who had stopped smoking before less than one year are considered current smokers in the analyses.

Table 2 shows multivariate logistic regression analyses of the cross-sectional associations between eating habits and age, living arrangement, financial difficulties, symptoms of depression, and education. Table 3 shows multivariate logistic regression analyses of the cross-sectional associations of "daily PA" and "sports weekly" with age, living arrangement, financial difficulties, depressive symptoms, education, and current smoking.

Table 4 shows multivariate linear regressions of the cross-sectional associations of each of five anthropometric adiposity indicators with eating habits and PA. Eating habits and PA are simultaneously included in the same model. Since residuals had skewed distributions, all adiposity indicators were log-transformed. All potential confounders included in the adjustment are shown in Table 4. Statistical analyses were performed using Stata 12 software (Stata Corp, College Station, TX).

\section{Results}

\section{Eating habits and PA}

The prevalence of underweight $\left(\mathrm{BMI}<18.5 \mathrm{~kg} / \mathrm{m}^{2}\right)$ and normal weight was $0 \%$ and $23 \%$ among men, respectively 3\% and 39\% among women. Overweight and obesity prevalence was $53 \%$ and $24 \%$ among men, $35 \%$ and $23 \%$ among women. Abdominal obesity prevalence was $45 \%$ (both sexes). The prevalence of normal weight central obesity $\left(\mathrm{BMI}<25.0 \mathrm{~kg} / \mathrm{m}^{2}\right.$ and $\mathrm{WC} \geq 102 \mathrm{~cm}$ for men, $\geq 88 \mathrm{~cm}$ for women) was $0.0 \%$ among men, and $2.2 \%(16 / 736)$ among women. The prevalence with $\mathrm{BMI}<25.0 \mathrm{~kg} / \mathrm{m}^{2}$ and $\mathrm{WHR} \geq 66$ th gender-specific percentile was $2.3 \%$ in men and $5.0 \%$ in women (Table 1 ).

According to Table 1, 81\% of men and $90 \%$ of women ate fruit or vegetables (FV) $\geq 2 \times /$ day (chi-square $P<0.001$ ). $5 \%$ of both men and women reported moderate appetite loss and 4\% moderate malnutrition (or unawareness of their own nutritional state).

Concerning physical activity (PA) in daily life, a higher proportion of women (25\%) avoided using stairs or carrying loads than men $(20 \%$, chi-square $P=0.003)$. About half of men and women played sports $<1 \times /$ week, without any significant sex difference. Women more frequently lived alone $(47 \%$ vs. $19 \%$, chi-square $P<0.001)$ and more often experienced symptoms of depression than men (28\% vs. 19\%, chi-square $P<0.001$ ). Financial difficulties (about one quarter) and smoking (about one fifth) were equally distributed among sexes.

\section{Links of eating habits and physical activity with socio-economic factors}

According to Table 2, eating FV $\geq$ twice/day was negatively associated with financial difficulties and positively with education. Among men, eating 3 meals per day was significantly negatively associated with financial difficulties $(\mathrm{OR}=0.6,95 \% \mathrm{CI}=0.4-0.9)$. Among women, eating meat, fish, or poultry every day was positively related with being younger $(\mathrm{OR}=1.1,95 \% \mathrm{CI}=1.0-1.2$ for each additional birth year); but very strongly negatively with living alone $(\mathrm{OR}=0.5,95 \% \mathrm{CI}=0.4-0.7)$. Men living alone were less likely to eat $\mathrm{FV} \geq 2 \times /$ day $(\mathrm{OR}=0.5,95 \%$ $\mathrm{CI}=0.3-0.9$ ). Self-perception of malnutrition was strongly associated with living alone in both sexes. In men, this perception was also associated with financial difficulties $(\mathrm{OR}=3.2,95 \% \mathrm{CI}=1.2-8.7)$. Symptoms of depression were strongly associated with appetite loss in both sexes and with perception of moderate malnutrition in women $(\mathrm{OR}=3.8,95 \% \mathrm{CI}=1.7-8.5)$.

Table 3 shows that both daily PA and sports weekly have strong and significant negative links with financial difficulties 
Table 2 Associations of eating habits with age, living arrangement, financial difficulties, symptoms of depression, and education

\begin{tabular}{|c|c|c|c|c|c|}
\hline & $\begin{array}{l}3 \text { meals/day } \\
\text { (versus } 1 \text { or } 2 \text { ) }\end{array}$ & $\begin{array}{l}\text { Dairy products } \geq \text { once a day } \\
\text { (yes versus no) }\end{array}$ & $\begin{array}{l}\text { Eggs or leguminous } \\
\text { plants } \geq \text { twice/week } \\
\text { (yes versus no) }\end{array}$ & $\begin{array}{l}\text { Meat, fish or poultry } \\
\text { everyday (yes versus no) }\end{array}$ & $\begin{array}{l}\text { "Sufficient protein } \\
\text { intake" } \\
\text { (yes versus no) }\end{array}$ \\
\hline & OR $[95 \% \mathrm{Cl}]$ & OR $[95 \% \mathrm{Cl}]$ & OR $[95 \% \mathrm{Cl}]$ & OR $[95 \% \mathrm{Cl}]$ & OR $[95 \% \mathrm{Cl}]$ \\
\hline \multicolumn{6}{|l|}{ MEN } \\
\hline Age (per 1-birth year) & $1.0[0.9-1.1]$ & $0.8[0.7-1.0]^{*}$ & $1.1[0.9-1.2]$ & $1.1[0.9-1.2]$ & $1.1[0.9-1.3]$ \\
\hline Living alone (0/1) & $0.7[0.5-1.2]$ & $0.7[0.4-1.2]$ & $0.8[0.5-1.3]$ & $0.9[0.6-1.5]$ & $0.9[0.5-1.5]$ \\
\hline Financial difficulties $(0 / 1)^{\dagger}$ & $0.6[0.4-0.9]^{*}$ & $0.7[0.4-1.1]$ & $0.9[0.6-1.4]$ & $0.8[0.5-1.2]$ & $0.7[0.5-1.1]$ \\
\hline Symptoms of depression (0/1) & $0.7[0.4-1.1]$ & $1.0[0.5-1.8]$ & $0.9[0.6-1.4]$ & $1.0[0.6-1.6]$ & $0.9[0.6-1.5]$ \\
\hline \multicolumn{6}{|l|}{ Education } \\
\hline Basic compulsory & 1.0 (ref) & 1.0 (ref) & 1.0 (ref) & 1.0 (ref) & 1.0 (ref) \\
\hline Apprenticeship & $1.0[0.6-1.7]$ & $2.0[1.1-3.7]^{*}$ & $1.6[1.0-2.8]$ & $1.6[0.9-2.7]$ & $1.9[1.1-3.4]^{*}$ \\
\hline High school or more & $1.4[0.8-2.3]$ & $2.6[1.4-4.9]^{* *}$ & $1.6[1.0-2.8]$ & $1.2[0.7-2.0]$ & $1.5[0.8-2.5]$ \\
\hline \multicolumn{6}{|l|}{ WOMEN } \\
\hline Age (per 1-birth year) & $1.0[0.9-1.1]$ & $0.9[0.8-1.1]$ & $0.9[0.8-1.0]$ & $1.1[1.0-1.2]^{*}$ & $1.0[0.9-1.1]$ \\
\hline Living alone (0/1) & $0.9[0.7-1.2]$ & $0.8[0.5-1.2]$ & $0.7[0.6-1.0]$ & $0.5[0.4-0.7]^{* * *}$ & $0.5[0.4-0.8]^{* * *}$ \\
\hline Financial difficulties $(0 / 1)^{\dagger}$ & $0.8[0.5-1.1]$ & $1.1[0.7-1.9]$ & $0.9[0.6-1.2]$ & $1.0[0.7-1.3]$ & $0.8[0.6-1.2]$ \\
\hline Symptoms of depression $(0 / 1)$ & $0.7[0.5-1.0]$ & $0.8[0.5-1.3]$ & $1.2[0.9-1.7]$ & $1.3[0.9-1.8]$ & $1.5[1.0-2.2]$ \\
\hline \multicolumn{6}{|l|}{ Education } \\
\hline Basic compulsory & 1.0 (ref) & 1.0 (ref) & 1.0 (ref) & 1.0 (ref) & 1.0 (ref) \\
\hline Apprenticeship & $0.9[0.6-1.3]$ & $1.4[0.8-2.5]$ & $1.2[0.9-1.8]$ & $1.0[0.7-1.4]$ & $1.1[0.7-1.6]$ \\
\hline \multirow[t]{3}{*}{ High school or more } & $1.2[0.8-1.8]$ & $1.0[0.6-1.7]$ & $1.1[0.7-1.6]$ & $0.8[0.6-1.2]$ & $0.9[0.6-1.3]$ \\
\hline & $\begin{array}{l}\text { Fruit or vegetables } \geq \\
\text { twice/day (yes versus no) }\end{array}$ & $\begin{array}{l}>5 \text { glasses/day } \\
\text { (versus } \leq 5 \text { drinks/day) }\end{array}$ & $\begin{array}{l}\text { Loss of appetite } \\
\text { (yes versus not at all) }\end{array}$ & $\begin{array}{l}\text { Self-perception: moderate } \\
\text { malnutrition or doesn't know } \\
\text { (versus no nutritional problems) }\end{array}$ & \\
\hline & OR [95\% Cl] & OR $[95 \% \mathrm{Cl}]$ & OR $[95 \% \mathrm{Cl}]$ & OR $[95 \% \mathrm{Cl}]$ & \\
\hline \multicolumn{6}{|l|}{ MEN } \\
\hline Age (per 1-birth year) & $1.0[0.9-1.2]$ & $1.1[0.9-1.2]$ & $1.0[0.8-1.4]$ & $1.0[0.7-1.5]$ & \\
\hline Living alone (0/1) & $0.5[0.3-0.9]^{*}$ & $1.0[0.6-1.6]$ & $2.2[0.9-5.4]$ & $5.0[1.9-13.6]^{* *}$ & \\
\hline Financial difficulties $(0 / 1)^{\dagger}$ & $0.6[0.4-1.0]^{*}$ & $1.0[0.7-1.6]$ & $1.1[0.4-2.8]$ & $3.2[1.2-8.7]^{*}$ & \\
\hline Symptoms of depression (0/1) & $0.7[0.4-1.3]$ & $0.9[0.6-1.4]$ & $2.5[1.1-6.1]^{*}$ & $1.6[0.5-4.5]$ & \\
\hline \multicolumn{6}{|l|}{ Education } \\
\hline Basic compulsory & 1.0 (ref) & 1.0 (ref) & 1.0 (ref) & 1.0 (ref) & \\
\hline Apprenticeship & $1.2[0.7-2.2]$ & $0.6[0.4-1.0]$ & $1.7[0.5-6.5]$ & $1.6[0.4-6.4]$ & \\
\hline High school or more & $2.4[1.2-4.5]^{*}$ & $0.6[0.4-1.0]$ & $1.0[0.3-4.1]$ & $0.8[0.2-3.5]$ & \\
\hline
\end{tabular}


Table 2 Associations of eating habits with age, living arrangement, financial difficulties, symptoms of depression, and education (Continued)

\begin{tabular}{|c|c|c|c|c|}
\hline WOMEN & & & & \\
\hline Age (per 1-birth year) & $1.0[0.9-1.2]$ & $1.2[1.0-1.3]^{*}$ & $1.0[0.8-1.3]$ & $1.1[0.8-1.5]$ \\
\hline Living alone $(0 / 1)$ & $0.8[0.5-1.4]$ & $0.8[0.6-1.1]$ & $1.1[0.6-2.1]$ & $2.5[1.1-5.9]^{*}$ \\
\hline Financial difficulties $(0 / 1)^{+}$ & $0.5[0.3-0.9]^{*}$ & $1.2[0.8-1.7]$ & $1.2[0.6-2.5]$ & $1.4[0.6-3.3]$ \\
\hline Symptoms of depression $(0 / 1)$ & $1.2[0.7-2.0]$ & $1.1[0.8-1.5]$ & $3.4[1.7-6.6]^{* * *}$ & $3.8[1.7-8.5]^{* *}$ \\
\hline \multicolumn{5}{|l|}{ Education } \\
\hline Basic compulsory & 1.0 (ref) & 1.0 (ref) & 1.0 (ref) & 1.0 (ref) \\
\hline Apprenticeship & $1.2[0.7-2.2]$ & $1.2[0.8-1.7]$ & $1.0[0.4-2.3]$ & $1.0[0.3-2.7]$ \\
\hline High school or more & $1.8[0.9-3.4]$ & $1.0[0.7-1.5]$ & $1.9[0.9-4.4]$ & $1.6[0.6-4.2]$ \\
\hline
\end{tabular}

Multivariate logistic regression analysis; one separate model for each sex; approximate $\mathrm{N}=507$ for men, 724 for women.

${ }^{*} \mathrm{P}<0.05 ; * * \mathrm{P}<0.01 ; * * \mathrm{*}<0.001$.

${ }^{\dagger}$ Financial difficulties were considered if any of the following criteria was fulfilled: 1) current income clearly lower than others, 2) sometimes difficulty to make ends meet, 3) subsidy for health insurance,

or 4) complementary subsidy (from old age insurance).

‡"Sufficient protein intake" was defined if the participant reported eating meat, fish or poultry every day, or as an alternative, if he consumed dairy products $\geq$ once a day and eggs or leguminous plants $\geq$ twice/week. 
Table 3 Association of physical activity with socio-economic and lifestyle factors

\begin{tabular}{|c|c|c|c|c|}
\hline & \multicolumn{2}{|c|}{ Daily PA } & \multicolumn{2}{|c|}{ Sports weekly } \\
\hline & OR $[95 \% \mathrm{Cl}]$ & $P$-value & OR $[95 \% \mathrm{Cl}]$ & $P$-value \\
\hline \multicolumn{5}{|l|}{ MEN } \\
\hline N & 501 & & 486 & \\
\hline Age (per 1-birth year) & $1.0[0.8-1.1]$ & 0.623 & $1.0[0.9-1.1]$ & 0.865 \\
\hline Living alone $(0 / 1)$ & $0.5[0.3-0.9]$ & 0.013 & $0.7[0.4-1.1]$ & 0.094 \\
\hline Financial difficulties $(0 / 1)^{\dagger}$ & $0.4[0.3-0.7]$ & 0.001 & $0.5[0.3-0.8]$ & 0.002 \\
\hline Symptoms of depression $(0 / 1)$ & $0.8[0.4-1.3]$ & 0.367 & $0.9[0.5-1.4]$ & 0.635 \\
\hline \multicolumn{5}{|l|}{ Education } \\
\hline Basic compulsory & 1 (ref) & & 1 (ref) & \\
\hline Apprenticeship & $1.1[0.6-2.1]$ & 0.780 & $1.0[0.6-1.7]$ & 0.991 \\
\hline High school or more & $1.6[0.8-3.1]$ & 0.150 & $1.4[0.8-2.4]$ & 0.265 \\
\hline Current smoking $(0 / 1)^{\ddagger}$ & $0.6[0.4-1.1]$ & 0.090 & $0.4[0.3-0.7]$ & $<0.001$ \\
\hline \multicolumn{5}{|l|}{ WOMEN } \\
\hline N & 713 & & 677 & \\
\hline Age (per 1-birth year) & $1.1[0.9-1.2]$ & 0.373 & $1.1[1.0-1.2]$ & 0.131 \\
\hline Living alone $(0 / 1)$ & $0.8[0.6-1.1]$ & 0.234 & $1.0[0.7-1.4]$ & 0.978 \\
\hline Financial difficulties $(0 / 1)^{\dagger}$ & $0.6[0.4-0.9]$ & 0.008 & $0.6[0.4-0.8]$ & 0.002 \\
\hline Symptoms of depression $(0 / 1)$ & $0.6[0.4-0.9]$ & 0.017 & $1.0[0.7-1.4]$ & 0.933 \\
\hline \multicolumn{5}{|l|}{ Education } \\
\hline Basic compulsory & 1 (ref) & & 1 (ref) & \\
\hline Apprenticeship & $1.2[0.8-1.8]$ & 0.386 & $1.2[0.8-1.7]$ & 0.473 \\
\hline High school or more & $1.7[1.1-2.7]$ & 0.018 & $1.7[1.1-2.6]$ & 0.010 \\
\hline Current smoking $(0 / 1)^{\ddagger}$ & $0.8[0.6-1.3]$ & 0.458 & $0.4[0.3-0.7]$ & $<0.001$ \\
\hline \multicolumn{5}{|l|}{ ALL } \\
\hline N & 1214 & & 1163 & \\
\hline Age (per 1-birth year) & $1.0[0.9-1.1]$ & 0.675 & $1.0[1.0-1.1]$ & 0.290 \\
\hline Male sex & $1.3[0.5-3.2]$ & 0.588 & $1.5[0.7-3.3]$ & 0.331 \\
\hline Living alone $(0 / 1)$ & $0.8[0.6-1.2]$ & 0.289 & $1.0[0.7-1.4]$ & 0.985 \\
\hline Financial difficulties $(0 / 1)^{\dagger}$ & $0.5[0.4-0.7]$ & $<0.001$ & $0.5[0.4-0.7]$ & $<0.001$ \\
\hline Symptoms of depression $(0 / 1)$ & $0.6[0.4-0.9]$ & 0.023 & $1.0[0.7-1.4]$ & 0.954 \\
\hline \multicolumn{5}{|l|}{ Education } \\
\hline Basic compulsory & 1 (ref) & & 1 (ref) & \\
\hline Apprenticeship & $1.2[0.8-1.7]$ & 0.414 & $1.1[0.8-1.6]$ & 0.443 \\
\hline High school or more & $1.7[1.1-2.7]$ & 0.021 & $1.7[1.1-2.6]$ & 0.009 \\
\hline Current smoking $(0 / 1)^{\ddagger}$ & $0.8[0.5-1.1]$ & 0.102 & $0.4[0.3-0.6]$ & $<0.001$ \\
\hline Living alone ${ }^{*}$ male sex & $0.6[0.3-1.1]$ & 0.115 & $0.7[0.4-1.2]$ & 0.151 \\
\hline Depressive symptoms*male sex & $1.1[0.6-2.2]$ & 0.687 & $0.9[0.5-1.6]$ & 0.691 \\
\hline Education*male sex & $1.0[0.7-1.5]$ & 0.951 & $0.9[0.6-1.3]$ & 0.528 \\
\hline
\end{tabular}

Daily physical activity (PA, 0/1):1) Often walking and using stairs, carrying light loads, and important physical activity, carries heavy loads; 0$)$ sitting or lying most of the time, or often walking, but avoiding stairs and loads. Sports weekly (0/1): Sports frequency $<1 \times /$ week (0), versus $\geq 1 \times /$ week (1). Multivariate logistic regression analysis.

${ }^{\dagger}$ Financial difficulties were considered if any of the following criteria was fulfilled: 1) current income clearly lower than others, 2) sometimes difficulty to make ends meet, 3) subsidy for health insurance, or 4) complementary subsidy (from old age insurance).

${ }^{\ddagger}$ Participants who had stopped smoking before less than one year are considered current smokers in the analyses.

*Interaction. 


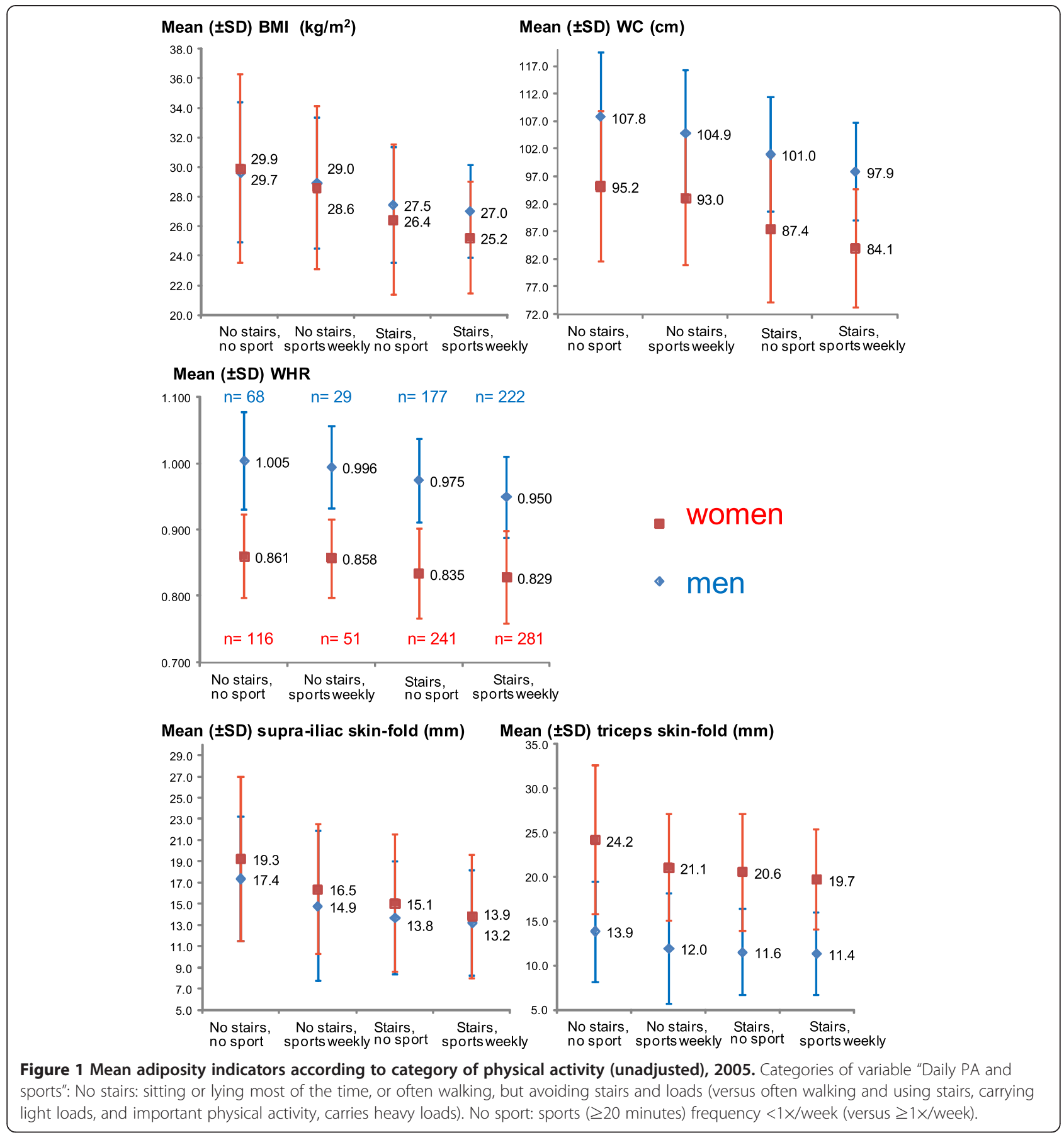

in both sexes, and significant positive links with educational level in women only; however the interaction was not statistically significant. Unlike women, men living alone were significantly less likely to use stairs and carry light loads in their daily life than men living with someone $(\mathrm{OR}=0.5$, 95\% CI $=0.3-0.9)$. No sex interaction reached significance. Sports weekly, but not daily PA, had strong significant negative links with current smoking in both sexes.

Across the 4 categories of variable "Daily PA and sports", there was a gradual decrease in the prevalence of participants living alone, experiencing financial difficulties, depressive symptoms, current smoking, and a gradual increase in educational level. The test for trend $P$-value was statistically significant $(P<0.05)$ for all above mentioned associations (Additional file 1).

Links of eating habits and physical activity with adiposity The Figure 1 shows univariate associations between each adiposity indicator and variable "Daily PA and sports". Men had higher BMI, WC, and waist-to-hip ratio (WHR) 
Table 4 Associations of five log-transformed adiposity indicators with eating habits and physical activity

\begin{tabular}{|c|c|c|c|c|c|c|c|c|c|c|}
\hline & Ln (BMI) & & $\operatorname{Ln}(W C)$ & & Ln (WHR) & & $\operatorname{Ln}(\mathrm{SISF})$ & & Ln (TSF) & \\
\hline & $B[95 \% \mathrm{Cl}]$ & $P$ & $B[95 \% \mathrm{Cl}]$ & $P$ & $B[95 \% \mathrm{Cl}]$ & $P$ & $B[95 \% \mathrm{Cl}]$ & $P$ & $B[95 \% \mathrm{Cl}]$ & $P$ \\
\hline \multicolumn{11}{|l|}{ MEN: $(N \geq 477)$ : } \\
\hline \multicolumn{11}{|l|}{ Eating habits: } \\
\hline Three meals/day & $-0.02[-0.04-0.01]$ & & $-0.01[-0.02-0.01]$ & & $-0.01[-0.02-0.00]$ & & $0.00[-0.07-0.08]$ & & $-0.04[-0.11-0.03]$ & \\
\hline Fruit and veg. $\geq$ twice/day & $0.00[-0.03-0.03]$ & & $0.00[-0.02-0.03]$ & & $0.00[-0.02-0.01]$ & & $0.05[-0.05-0.14]$ & & $0.02[-0.07-0.12]$ & \\
\hline Sufficient protein intake $\$$ & $0.03[0.00-0.06]$ & * & $0.03[0.01-0.05]$ & $* *$ & $0.02[0.01-0.03]$ & $* *$ & $0.04[-0.04-0.12]$ & & $-0.03[-0.11-0.04]$ & \\
\hline \multicolumn{11}{|l|}{ Daily PA and sports } \\
\hline 1: No stairs, no sport & 0 (ref.) & & 0 (ref.) & & 0 (ref.) & & 0 (ref.) & & 0 (ref.) & \\
\hline 2: No stairs, sports $\geq 1 \times /$ wk & $-0.03[-0.08-0.03]$ & & $-0.03[-0.07-0.02]$ & & $0.00[-0.03-0.03]$ & & $-0.25[-0.43--0.07]$ & $* *$ & $-0.22[-0.40--0.04]$ & * \\
\hline 3: Stairs, no sport & $-0.07[-0.11--0.04]$ & $* * *$ & $-0.06[-0.09--0.03]$ & $* * *$ & $-0.02[-0.04--0.01]$ & * & $-0.24[-0.35--0.13]$ & $* * *$ & $-0.18[-0.29--0.07]$ & $* *$ \\
\hline 4: Stairs, sports $\geq 1 \times /$ wk & $-0.09[-0.13--0.05]$ & $* * *$ & $-0.10[-0.12--0.07]$ & $* * *$ & $-0.05[-0.07--0.03]$ & $* * *$ & $-0.30[-0.41--0.18]$ & $* * *$ & $-0.20[-0.31--0.09]$ & $* *$ \\
\hline Age (per 1-birth year) & $0.01[0.00-0.01]$ & & $0.00[0.00-0.01]$ & & $0.00[0.00-0.01]$ & & $0.02[0.00-0.05]$ & & $0.00[-0.02-0.03]$ & \\
\hline Living alone $(0 / 1)$ & $-0.03[-0.06-0.00]$ & & $-0.01[-0.04-0.01]$ & & $0.01[-0.01-0.02]$ & & $0.03[-0.07-0.12]$ & & $0.02[-0.08-0.11]$ & \\
\hline Financial diff. $(0 / 1)^{€}$ & $-0.01[-0.03-0.02]$ & & $0.00[-0.02-0.02]$ & & $0.00[-0.01-0.01]$ & & $-0.01[-0.10-0.08]$ & & $0.01[-0.07-0.10]$ & \\
\hline Symptoms of depression & $0.03[0.00-0.06]$ & * & $0.02[-0.01-0.04]$ & & $0.00[-0.01-0.02]$ & & $0.06[-0.04-0.15]$ & & $0.04[-0.06-0.13]$ & \\
\hline \multicolumn{11}{|l|}{ Education } \\
\hline Basic compulsory & 0 (ref.) & & 0 (ref.) & & 0 (ref.) & & 0 (ref.) & & 0 (ref.) & \\
\hline Apprenticeship & $-0.03[-0.07-0.00]$ & & $-0.02[-0.05-0.01]$ & & $-0.01[-0.03-0.01]$ & & $-0.01[-0.11-0.10]$ & & $-0.03[-0.13-0.07]$ & \\
\hline$\geq$ High school & $-0.05[-0.09--0.02]$ & $* *$ & $-0.03[-0.06--0.01]$ & * & $-0.02[-0.04--0.01]$ & * & $-0.02[-0.13-0.08]$ & & $-0.03[-0.13-0.08]$ & \\
\hline Current smoking $(0 / 1) \S$ & $-0.04[-0.07--0.01]$ & * & $-0.01[-0.03-0.01]$ & & $0.01[-0.01-0.02]$ & & $-0.11[-0.20--0.03]$ & * & $-0.05[-0.14-0.03]$ & \\
\hline
\end{tabular}

Eating habits:

\begin{tabular}{|c|c|c|c|c|c|c|c|}
\hline Three meals/day & $0.00[-0.03-0.03]$ & & $0.01[-0.02-0.03]$ & $0.00[-0.01-0.01]$ & $0.06[-0.02-0.13]$ & & $-0.03[-0.09-0.03]$ \\
\hline Fruit and veg. $\geq$ twice/day & $0.05[0.00-0.10]$ & $*$ & $0.02[-0.02-0.05]$ & $-0.01[-0.03-0.01]$ & $0.14[0.02-0.27]$ & * & $0.14[0.04-0.23]$ \\
\hline Sufficient protein intake & $0.03[0.00-0.06]$ & & 0.02 [0.00-0.04] & $0.01[-0.01-0.02]$ & $0.04[-0.04-0.11]$ & & $0.05[-0.01-0.11]$ \\
\hline
\end{tabular}

Daily PA and sports

\begin{tabular}{|c|c|c|c|c|c|c|c|c|c|c|}
\hline 1: No stairs, no sport & 0 (ref.) & & 0 (ref.) & & 0 (ref.) & & 0 (ref.) & & 0 (ref.) & \\
\hline 2: No stairs, sports $\geq 1 \times /$ wk & $-0.04[-0.10-0.01]$ & & $-0.02[-0.07-0.02]$ & & $0.00[-0.03-0.03]$ & & $-0.16[-0.32-0.00]$ & * & $-0.13[-0.25--0.01]$ & * \\
\hline 3: Stairs, no sport & $-0.12[-0.16--0.08]$ & $* * *$ & $-0.08[-0.11--0.05]$ & $* * *$ & $-0.03[-0.04--0.01]$ & $* *$ & $-0.25[-0.36--0.14]$ & $* * *$ & $-0.15[-0.23--0.07]$ & $* * *$ \\
\hline 4: Stairs, sports $\geq 1 \times /$ wk & $-0.16[-0.20--0.12]$ & $* * *$ & $-0.12[-0.15--0.09]$ & $* * *$ & $-0.03[-0.05--0.01]$ & $* *$ & $-0.34[-0.45--0.23]$ & $* * *$ & $-0.19[-0.27--0.11]$ & *** \\
\hline (per 1-birth year) & $0.00[-0.01-0.01]$ & & $0.00[-0.01-0.01]$ & & $0.00[0.00-0.00]$ & & $0.01[-0.02-0.03]$ & & $0.00[-0.02-0.02]$ & \\
\hline iving alone $(0 / 1)$ & $-0.03[-0.06-0.00]$ & * & $-0.02[-0.04-0.00]$ & & $-0.01[-0.02-0.01]$ & & $-0.05[-0.12-0.03]$ & & $-0.06[-0.11-0.00]$ & * \\
\hline inancial diff. $(0 / 1)^{€}$ & $0.04[0.01-0.07]$ & * & $0.02[-0.01-0.04]$ & & $0.00[-0.01-0.02]$ & & $0.08[-0.01-0.16]$ & & $0.05[-0.01-0.12]$ & \\
\hline ymptoms of depression & $0.01[-0.02-0.04]$ & & $0.01[-0.01-0.04]$ & & $0.01[0.00-0.02]$ & & $0.06[-0.02-0.14]$ & & $0.01[-0.05-0.07]$ & \\
\hline \multicolumn{11}{|l|}{ ducation } \\
\hline Basic compulsory & 0 (ref.) & & 0 (ref.) & & 0 (ref.) & & 0 (ref.) & & 0 (ref.) & \\
\hline Apprenticeship & $-0.02[-0.05-0.01]$ & & $-0.01[-0.03-0.02]$ & & $-0.01[-0.02-0.01]$ & & $-0.04[-0.13-0.05]$ & & $-0.02[-0.09-0.05]$ & \\
\hline$\geq$ High school & $-0.06[-0.10--0.03]$ & *** & $-0.04[-0.06--0.01]$ & $* *$ & $-0.02[-0.03-0.00]$ & * & $-0.14[-0.23--0.05]$ & $* *$ & $-0.07[-0.14-0.00]$ & \\
\hline ent smoking $(0 / 1)^{\S}$ & $-0.07[-0.11--0.04]$ & $* * *$ & $-0.04[-0.06--0.01]$ & $* *$ & $0.00[-0.01-0.02]$ & & $-0.21[-0.30--0.12]$ & $* * *$ & $-0.15[-0.22--0.08]$ & \\
\hline
\end{tabular}

Multivariate linear regression analysis. The table shows all covariates included in the adjustment.

\$ "Sufficient protein intake" was defined if the participant reported eating meat, fish or poultry every day, or as an alternative, if he consumed dairy products $\geq$ once a day and eggs or leguminous plants $\geq$ twice/week.

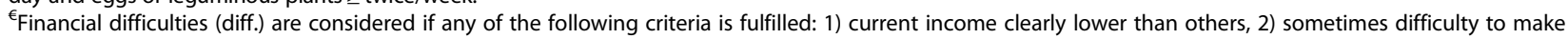
ends meet, 3) subsidy for health insurance, or 4) complementary subsidy (from old age insurance).

${ }^{\S}$ Participants who had stopped smoking before less than one year are considered current smokers in the analyses.

${ }^{*} \mathrm{P}<0.05 ;{ }^{* *} \mathrm{P}<0.01$; ${ }^{* * *} \mathrm{P}<0.001$. 
mean values than women. In contrary, women had higher SISF and TSF mean values than men. For all five adiposity indicators and in both sexes, a progressive decrease in adiposity was observed from category "No stairs, no sport", to "No stairs, sports weekly", to "Stairs, no sport", and finally "Stairs, sports weekly". This consistent univariate association was statistically significant for all indicators (test for trend $P$-value $<0.001$ for all indicators, except TSF among men: $P$-value $=0.002$ ). This Figure 1 suggests that adiposity values were higher among participants taking no stairs in daily life, but doing sports $\geq 1 \times /$ week, than among persons taking stairs, but doing sports $<1 \times \mid$ week. The same progressive decrease in median adiposity values across categories of PA was observed for all five indicators and in both sexes (Additional file 2). The prevalence of obesity (BMI $\geq 30.0 \mathrm{~kg} / \mathrm{m}^{2}$ ) was respectively $45.6 \%, 41.4 \%, 19.3 \%$, and $17.6 \%$ in men, respectively $44.8 \%, 41.2 \%, 21.6 \%$, and $11.0 \%$ in women in categories "no stairs, sports $<$ once/week", "no stairs, sports $\geq$ once/week", "stairs, sports $<$ once/week", and "stairs, sports $\geq$ once/ week" (chi-square and univariate test for trend $P$ values $<0.001$ in both sexes). Corresponding prevalence estimates for abdominal obesity were $69.7 \%, 62.1 \%, 44.0 \%$, and $34.2 \%$ in men, respectively $69.0 \%, 68.6 \%, 42.3 \%$, and $32.7 \%$ in women (chi-square and univariate test for trend $P$-values $<0.001$ in both sexes).

According to Table 4, "sufficient protein intake" was positively linked with BMI, WC, and WHR in men. FV intake was positively related to BMI, SISF, and TSF in women. Except for SISF and TSF in men (with similar $\beta$ coefficients across PA categories), the gradual decrease in adiposity across categories of increasing PA observed in the Figure 1 was confirmed with multiple adjustment in both sexes (Table 4). Unlike men, women living alone had slightly lower BMI and TSF values than women living with someone. Current smoking was strongly significantly negatively associated with all adiposity indicators but WHR in women. Additional adjustment for number of chronic diseases and self-rated health yielded similar results (Additional file 3).

\section{Discussion}

In this community-dwelling population aged 65-70, overweight and obesity affected more than three quarters of men, and between half and two thirds of women. Abdominal obesity almost hit half of both sexes. Normal weight abdominal obesity, defined as $\mathrm{BMI}<25 \mathrm{~kg} / \mathrm{m}^{2}$ and WC $\geq 102 \mathrm{~cm}$ in men, $88 \mathrm{~cm}$ in women, was absent among men, and very rare among women. A gradual decrease in adiposity was almost consistently observed across categories of increasing PA. Adiposity values were higher among participants taking no stairs in daily life, but doing sports $\geq 1 \times /$ week, than among persons taking stairs, but doing sports $<1 \times /$ week. In both sexes, eating
FV $\geq 2 \times /$ day, taking stairs every day, and doing sports $\geq$ once a week were strongly negatively associated with financial difficulties, and positively with education. The independent and significant negative association of adiposity with PA and education in Lc65+ might be explained by higher fat and total energy intake among less educated persons [33]; these nutritional items were not assessed. A large European cross-sectional study reported a strong association between low levels of PA (during work and leisure time) and obesity, while adjusting for educational level and total energy intake [34].

The main limitations of the present study include the lack of data on total energy intake, fat intake, and sugar intake (the MNA does not include any question on dessert or sugar); the lack of information on total energy intake limited the interpretation of the results, in particular for Table 4. MNA items are categorical and do not allow any quantitative estimation of food intake. Therefore, the present report only presents a few items about eating habits, and does not provide a real dietary assessment. Daily PA and sports frequency were selfreported; a measurement bias could have occurred if participants with higher adiposity values had over-reported their sports frequency, but not their daily PA. To our knowledge, no such a systematic differential bias has previously been described. These analyses could be replicated in studies objectively measuring daily PA and sports frequency. The number of stairs was not specified in the question asked to the participants, nor the number of minutes spent on each of the sports (except that it was at least 20 minutes per session). Therefore, it is not precise enough to clearly establish that there is a dose-response relationship. In addition, the specific benefits of walking and using stairs could not be precisely separated and compared, since a single question addressed both these activities. The sample size was relatively small, thus limiting the generalizability of the findings. This analysis should be replicated in larger samples. The present analysis was cross-sectional: low PA could lead to adiposity [35,36], in the same way as adiposity could lead to low PA $[37,38]$. The relationship between PA and adiposity should be studied longitudinally. A Swiss study observed that encouraging hospital employees to use stairs instead of elevators during their daily work routine significantly improved cardio-vascular disease risk factors (including WC, body weight, and fat mass) and increased cardiorespiratory fitness after 12 weeks [35]. On the other hand, a small study (involving adults younger than 65 years) reported that experimental weight gain (with overfeeding) reduced objectively measured daily walking distance [37]. In another longitudinal study [38], weight, BMI, fat mass, and WC predicted sedentary time after 5.6 years of follow-up, whereas sedentary time did not predict obesity. With increasing age, visceral abdominal fat mass increases 
$[4,8]$ while subcutaneous fat mass decreases. Increases in fat mass might not be reflected in proportional increases in anthropometric indicators [4]. However, according to Flegal et al. [39], BMI and WC may be inaccurate measures of percentage body fat for an individual, but they correspond well overall with percentage body fat within sex-age groups and distinguish categories of percentage body fat.

Strengths of Lc65+ include the randomly selected sample, which is representative of the general communitydwelling population of Lausanne aged 65 to 70. Its age distribution is homogeneous, reducing the potential for age-related biases (e.g. selection bias) or confounders. This cohort study offers a detailed description of the socioeconomic circumstances of this age group, which deserves careful attention for planning health services for the next decade. Height and weight were measured. Moreover, five anthropometric adiposity indicators were assessed, allowing examining the consistency of associations.

Lc65+ obesity prevalence estimates are slightly higher than those observed in the same city in CoLaus study [2], with a prevalence of overweight, obesity, and abdominal obesity of $50 \%, 23 \%$, and $40 \%$ in men aged $65-75$ years, respectively $35 \%, 17 \%$ and $45 \%$ in women (same definitions in CoLaus and Lc65+). In the Swiss Health Survey (SHS) 2012 [3], the prevalence of overweight and obesity (self-reported height and weight) was $49 \%$ and $17 \%$ in men, respectively $34 \%$ and $14 \%$ in women aged $65-74$ years at a national level. However, BMI calculated from selfreported height and weight is underestimated [40,41]. Studies allowing reliable international comparisons of overweight and obesity prevalence after age 65 years are lacking because of methodological issues (samples not always representative of the general population, differing participation rates, heterogeneous age distributions, measurements versus self-reports of weight and height) [42]. Despite these limitations, several reports suggest that overweight and obesity prevalence among Swiss adults is lower than in other European countries [40-42]. Several studies have described associations between low socioeconomic status and suboptimal diet [12] and PA [13,43], as well as the links of these behaviors with odds for being overweight or obese [44]. In Lc65+, living alone was associated with eating less FV among men, but less meat among women, a finding already observed in the SHS, and in England [45]. Lc65+ women living alone were also leaner; in a Swedish cohort [46], women "co-habitating" experienced a higher increase in weight and body fat since age 20 . Current smoking was consistently negatively associated with adiposity among Lc65+ women, a relationship also observed in CoLaus [2]. As regards daily PA, it has already been observed that pedestrians in lower socioeconomic areas are less likely to climb stairs and more often choose escalators than pedestrians in high socioeconomic areas [43]. Still, a stair climbing intervention was equally effective in both areas [43].

\section{Conclusions}

While the prevalence of abdominal obesity was $45 \%$ in both sexes, indicating an important risk of cardiovascular disease [47], $20 \%$ of men and $25 \%$ of women avoided using stairs or carrying loads. Whereas obese older persons should be encouraged to practice sports more than once a week [10], the importance of keeping a high level of mobility in daily life should not be overlooked [35]. In this population, eating habits and PA had strong links with socioeconomic factors, which could be the target of public health interventions [43]. Simple measures about use of stairs in everyday life can provide interesting information on health behaviors. In the present study, these measures had consistent cross-sectional associations with adiposity indicators. In conclusion, this study suggests that walking and using stairs in daily life has stronger negative links with adiposity than doing sports at least once a week.

\section{Ethics approval}

The ethics committee of the Faculty of Biology and Medicine of the University of Lausanne has approved the study protocol.

\section{Additional files}

Additional file 1: Associations between variable "Daily PA and sports" and socio-economic factors and lifestyle factors.

Additional file 2: Median values of the 5 adiposity indicators according to variable "Daily PA and sports".

Additional file 3: Associations of adiposity with diet and physical activity, adjusted for number of chronic diseases. Associations of adiposity with diet and physical activity, adjusted for self-rated health.

\section{Abbreviations}

BMI: Body mass index; Cl: Confidence intervals; FV: Fruits or vegetables; MNA: Mini nutritional assessment; OR: Odds ratio; PA: Physical activity; SHS: Swiss Health Survey; SISF: Supra-iliac skin-fold; TSF: Triceps skin-fold; WC: Waist circumference; WHR: Waist-to-hip ratio.

\section{Competing interests}

The authors declare that they have no competing interests.

\section{Authors' contributions}

Study design: SE. Data acquisition: SE. Data analysis and interpretation: DH, SE. Manuscript preparation: DH, SE. Both authors read and approved the final manuscript.

\section{Acknowledgements}

The data used in this manuscript stem from the Lc65+ cohort study, a project exclusively funded by public institutions. Since 2004, the Lc65+ project has been funded by the Swiss National Foundation for Scientific Research [3247B0-120795/1]; the Faculty of Biology and Medicine, University of Lausanne; the Loterie Romande (a non-profit organization supporting research and social projects); the Fondation Médecine Sociale et Préventive, Lausanne; the University of Lausanne Hospital Centre and its Institute of 
Social and Preventive Medicine; the Service of Geriatric Medicine and Geriatric Rehabilitation; the University of Lausanne Department of Ambulatory Care and Community Medicine; the Canton de Vaud Department of Public Health; and the City of Lausanne. Nadia Danon-Hersch has received a Marie-Heim Vögtlin subsidy from the Swiss National Science Foundation for a position as a PhD candidate for 2 years.

The authors thank all Lc65+ participants for their involvement in the study. We are grateful to the medical assistants who conducted the tests at the study center and to those who assisted us in data acquisition

Received: 28 June 2013 Accepted: 5 December 2013

Published: 13 December 2013

\section{References}

1. Marques-Vidal P, Bovet P, Paccaud F, Chiolero A: Changes of overweight and obesity in the adult Swiss population according to educational level, from 1992 to 2007. BMC Public Health 2010, 10:87.

2. Marques-Vidal P, Bochud M, Mooser V, Paccaud F, Waeber G, Vollenweider $P$ : Prevalence of obesity and abdominal obesity in the Lausanne population. BMC Public Health 2008, 8:330.

3. Body mass index weight categories, Swiss Health Survey 2012. http:// www.bfs.admin.ch/bfs/portal/fr/index/themen/14/02/02/key/02.html.

4. Zamboni M, Mazzali G: Obesity in the elderly: an emerging health issue. Int J Obes (Lond) 2012, 36:1151-1152.

5. Danon-Hersch N, Rodondi N, Spagnoli J, Santos-Eggimann B: Prefrailty and chronic morbidity in the youngest old: an insight from the Lausanne cohort Lc65+. J Am Geriatr Soc 2012, 60:1687-1694.

6. Cruz-Jentoft AJ, Baeyens JP, Bauer JM, Boirie Y, Cederholm T, Landi F, Martin FC, Michel JP, Rolland Y, Schneider SM, et al: Sarcopenia: European consensus on definition and diagnosis: Report of the European Working Group on Sarcopenia in Older People. Age Ageing 2010, 39:412-423.

7. Stenholm S, Alley D, Bandinelli S, Griswold ME, Koskinen S, Rantanen T, Guralnik JM, Ferrucci L: The effect of obesity combined with low muscle strength on decline in mobility in older persons: results from the InCHIANTI study. Int J Obes (Lond) 2009, 33:635-644.

8. Atlantis E, Martin SA, Haren MT, Taylor AW, Wittert GA, Florey Adelaide Male Aging S: Lifestyle factors associated with age-related differences in body composition: the Florey Adelaide Male Aging Study. Am J Clin Nutr 2008, 88:95-104.

9. Zhang X, Shu XO, Xiang YB, Yang G, Li H, Gao J, Cai H, Gao YT, Zheng W: Cruciferous vegetable consumption is associated with a reduced risk of total and cardiovascular disease mortality. Am J Clin Nutr 2011, 94:240-246.

10. Villareal DT, Chode S, Parimi N, Sinacore DR, Hilton T, Armamento-Villareal R, Napoli N, Qualls C, Shah K: Weight loss, exercise, or both and physical function in obese older adults. N Engl J Med 2011, 364:1218-1229.

11. American College of Sports M, Chodzko-Zajko WJ, Proctor DN, Fiatarone Singh MA, Minson CT, Nigg CR, Salem GJ, Skinner JS: American College of Sports Medicine position stand. Exercise and physical activity for older adults. Med Sci Sports Exerc 2009, 41:1510-1530.

12. Watt $H C$, Carson C, Lawlor DA, Patel R, Ebrahim S: Influence of life course socioeconomic position on older women's health behaviors: findings from the British Women's Heart and Health Study. Am J Public Health 2009, 99:320-327.

13. Chad KE, Reeder BA, Harrison EL, Ashworth NL, Sheppard SM, Schultz SL, Bruner BG, Fisher KL, Lawson JA: Profile of physical activity levels in community-dwelling older adults. Med Sci Sports Exerc 2005, 37:1774-1784

14. de Abreu D, Guessous I, Vaucher J, Preisig M, Waeber G, Vollenweider P, Marques-Vidal P: Low compliance with dietary recommendations for food intake among adults. Clin Nutr 2013, 32:783-8.

15. Hendrie GA, Coveney J, Cox D: Exploring nutrition knowledge and the demographic variation in knowledge levels in an Australian community sample. Public Health Nutr 2008, 11:1365-1371.

16. Beydoun MA, Powell LM, Wang Y: The association of fast food, fruit and vegetable prices with dietary intakes among US adults: is there modification by family income? Soc Sci Med 2008, 66:2218-2229.

17. Poehlman ET, Toth MJ, Bunyard LB, Gardner AW, Donaldson KE, Colman E, Fonong T, Ades PA: Physiological predictors of increasing total and central adiposity in aging men and women. Arch Intern Med 1995, 155:2443-2448
18. Walls HL, Stevenson CE, Mannan HR, Abdullah A, Reid CM, McNeil J, Peeters A: Comparing trends in BMI and waist circumference. Obesity (Silver Spring) 2011, 19:216-219.

19. Santos-Eggimann B, Karmaniola A, Seematter-Bagnoud L, Spagnoli J, Bula C, Cornuz J, Rodondi N, Vollenweider P, Waeber G, Pecoud A: The Lausanne cohort Lc65+: a population-based prospective study of the manifestations, determinants and outcomes of frailty. BMC Geriatr 2008, 8:20.

20. Chen $X$, Huang $Y$, Cheng HG: Lower intake of vegetables and legumes associated with cognitive decline among illiterate elderly Chinese: a 3-year cohort study. J Nutr Health Aging 2012, 16:549-552.

21. Folstein MF, Folstein SE, McHugh PR: "Mini-mental state": a practical method for grading the cognitive state of patients for the clinician. J Psychiatr Res 1975, 12:189-198.

22. Vellas $B$, Villars $H$, Abellan $G$, Soto ME, Rolland $Y$, Guigoz $Y$, Morley JE, Chumlea W, Salva A, Rubenstein LZ, Garry P: Overview of the MNA-lts history and challenges. J Nutr Health Aging 2006, 10:456-463.

23. Wietlisbach V, Paccaud F, Rickenbach M, Gutzwiller F: Trends in cardiovascular risk factors (1984-1993) in a Swiss region: results of three population surveys. Prev Med 1997, 26:523-533.

24. Sequeira MM, Rickenbach M, Wietlisbach V, Tullen B, Schutz Y: Physical activity assessment using a pedometer and its comparison with a questionnaire in a large population survey. Am J Epidemiol 1995, 142:989-999.

25. Heymsfield SB, Heo M, Pietrobelli A: Are adult body circumferences associated with height? Relevance to normative ranges and circumferential indexes. Am J Clin Nutr 2011, 93:302-307.

26. Lean ME, Han TS, Morrison CE: Waist circumference as a measure for indicating need for weight management. BMJ 1995, 311:158-161.

27. Kaplan GA, Haan MN, Wallace RB: Understanding changing risk factor associations with increasing age in adults. Annu Rev Public Health 1999, 20:89-108.

28. Payne ME, Steck SE, George RR, Steffens DC: Fruit, vegetable, and antioxidant intakes are lower in older adults with depression. J Acad Nutr Diet 2012, 112:2022-2027.

29. Dvorak RD, Del Gaizo AL, Engdahl RM, Eliason CJ: Tobacco use and body mass index: mediated effects through physical inactivity. J Health Psychol 2009, 14:919-923.

30. Arroll B, Khin N, Kerse N: Screening for depression in primary care with two verbally asked questions: cross sectional study. BMJ 2003, 327:1144-1146.

31. Ledikwe JH, Smiciklas-Wright H, Mitchell DC, Jensen GL, Friedmann JM, Still CD: Nutritional risk assessment and obesity in rural older adults: a sex difference. Am J Clin Nutr 2003, 77:551-558.

32. Wu CH, Yao WJ, Lu FH, Yang YC, Wu JS, Chang CJ: Sex differences of body fat distribution and cardiovascular dysmetabolic factors in old age. Age Ageing 2001, 30:331-336.

33. Beydoun MA, Wang Y: How do socio-economic status, perceived economic barriers and nutritional benefits affect quality of dietary intake among US adults? Eur J Clin Nutr 2008, 62:303-313.

34. Besson H, Ekelund U, Luan J, May AM, Sharp S, Travier N, Agudo A, Slimani N, Rinaldi S, Jenab M, et al: A cross-sectional analysis of physical activity and obesity indicators in European participants of the EPIC-PANACEA study. Int J Obes (Lond) 2009, 33:497-506.

35. Meyer P, Kayser B, Kossovsky MP, Sigaud P, Carballo D, Keller PF, Martin XE, Farpour-Lambert N, Pichard C, Mach F: Stairs instead of elevators at workplace: cardioprotective effects of a pragmatic intervention. Eur J Cardiovasc Prev Rehabil 2010, 17:569-575.

36. Ekelund U, Besson H, Luan J, May AM, Sharp SJ, Brage S, Travier N, Agudo A, Slimani N, Rinaldi $S$, et al: Physical activity and gain in abdominal adiposity and body weight: prospective cohort study in 288,498 men and women. Am J Clin Nutr 2011, 93:826-835.

37. Levine JA, McCrady SK, Lanningham-Foster LM, Kane PH, Foster RC, Manohar $\mathrm{CU}$ : The role of free-living daily walking in human weight gain and obesity. Diabetes 2008, 57:548-554.

38. Ekelund U, Brage S, Besson H, Sharp S, Wareham NJ: Time spent being sedentary and weight gain in healthy adults: reverse or bidirectional causality? Am J Clin Nutr 2008, 88:612-617.

39. Flegal KM, Shepherd JA, Looker AC, Graubard BI, Borrud LG, Ogden CL, Harris TB, Everhart JE, Schenker N: Comparisons of percentage body fat, body mass index, waist circumference, and waist-stature ratio in adults. Am J Clin Nutr 2009, 89:500-508. 
40. Peytremann-Bridevaux I, Faeh D, Santos-Eggimann B: Prevalence of overweight and obesity in rural and urban settings of 10 European countries. Prev Med 2007, 44:442-446.

41. Berghofer A, Pischon T, Reinhold T, Apovian CM, Sharma AM, Willich SN: Obesity prevalence from a European perspective: a systematic review. BMC Public Health 2008, 8:200.

42. Haftenberger M, Lahmann PH, Panico S, Gonzalez CA, Seidell JC, Boeing H, Giurdanella MC, Krogh V, Bueno-de-Mesquita HB, Peeters PH, et al: Overweight, obesity and fat distribution in 50- to 64-year-old participants in the European Prospective Investigation into Cancer and Nutrition (EPIC). Public Health Nutr 2002, 5:1147-1162.

43. Ryan J, Lyon K, Webb OJ, Eves FF, Ryan CG: Promoting physical activity in a low socioeconomic area: results from an intervention targeting stair climbing. Prev Med 2011, 52:352-354.

44. Meyer AM, Evenson KR, Couper DJ, Stevens J, Pereria MA, Heiss G: Television, physical activity, diet, and body weight status: the ARIC cohort. Int J Behav Nutr Phys Act 2008, 5:68.

45. Donkin AJ, Johnson AE, Morgan K, Neale RJ, Page RM, Silburn RL: Gender and living alone as determinants of fruit and vegetable consumption among the elderly living at home in urban Nottingham. Appetite 1998, 30:39-51.

46. Lahmann PH, Lissner L, Gullberg B, Berglund G: Sociodemographic factors associated with long-term weight gain, current body fatness and central adiposity in Swedish women. Int I Obes Relat Metab Disord 2000, 24:685-694.

47. Zhang C, Rexrode KM, van Dam RM, Li TY, Hu FB: Abdominal obesity and the risk of all-cause, cardiovascular, and cancer mortality: sixteen years of follow-up in US women. Circulation 2008, 117:1658-1667.

doi:10.1186/1471-2458-13-1175

Cite this article as: Danon-Hersch and Santos-Eggimann: Physical activity in daily life is associated with lower adiposity values than doing weekly sports in Lc65+ cohort at baseline. BMC Public Health 2013 13:1175.

\section{Submit your next manuscript to BioMed Central and take full advantage of:}

- Convenient online submission

- Thorough peer review

- No space constraints or color figure charges

- Immediate publication on acceptance

- Inclusion in PubMed, CAS, Scopus and Google Scholar

- Research which is freely available for redistribution 